Erschienen in: Stickel, Gerhard (Hrsg.): Deutsche Gegenwartssprache. Tendenzen und Perspektiven. - Berlin, New York: de Gruyter, 1990. S. 21-29. (Institut für deutsche Sprache. Jahrbuch 1989)

Ansprache des Präsidenten des Instituts für deutsche Sprache

\author{
Prof. Dr. Siegfried Grosse
}

\title{
25 Jahre Institut für deutsche Sprache
}

Das Institut für Deutsche Sprache wird 198925 Jahre alt. Auf der vergeblichen Suche nach einer Erklärung für den Symbolgehalt der $25 \mathrm{kam}$ mir der Gedanke, man könne bei der Potenzierung der Heiligen Zahl 5 vielleicht zum einen an die 5 Finger der Hand denken und zum anderen an die 5 Sinne, dann ergäbe die fiktive Multiplikation eine besonders enge Verbindung und gegenseitige Steigerung von fleißigen Handfertigkeiten und wachem Verstandeswirken, was im Laufe von 25 Jahren wie man am Beispiel des Instituts sieht und seinen etwa 250 publizierten Büchern - zu durchaus respektablen Ergebnissen führen kann. Aber ich fürchte, daß ich mit diesem kläglichen Versuch einer zahlensymbolischen Deutung alle Interpretationsschwächen bloßgelegt habe, die Gefahr und Reiz jeder Textwissenschaft sind.

Die 25 scheint zumindest für Institutionen die kleinste festwürdige Zeitspanne zu sein, die man zu bekränzen und zu versilbern plegt. Dabei verlassen wir in der Zählung das uns so vertraute Dezimalsystem nur scheinbar, denn eine bei 25 eingelegte kurze Rast hat natürlich die noch weit entfernte $100 \mathrm{im}$ Visier, von der man immerhin ein Viertel geschafft hat. Der 100 gegenüber ist die 25 zwar nur ein kleiner Teil, doch gemessen an der Tätigkeit eines Hochschullehrers, die in der vollen Verantwortung des Dienstes meist erst mit dem 40. Lebensjahr beginnt und häufig schon beim 65. endet, ist sie alles. Eine solche Hochschullehrergeneration ist das Institut für Deutsche Sprache alt geworden, und mit ihm - ich spreche diese Selbstverständlichkeit nur aus, weil sie von manchen ignoriert wird - auch die Linguistik und die Gegenwartssprache selbst. Ich meine damit: auch wenn das Verständnis im Institut für den Plan, die Forschungen auf die Zeit vor 1945 auszudehnen, noch wachsen muß: wir alle - Institut und lebendes Inventar - müssen an einem solchen Besinnungstag wie heute feststellen, daß aus der Synchronie unserer Zukunft sehr schnell die Diachronie unserer Vergangenheit geworden ist.

Kuratorium, Vorstand und Institutsrat wollen dieses Jubiläum nicht mit affirmativen Lobeshymnen, Lorbeerbüschen und einem Streichquartett begehen, sondern wir möchten die Gelegenheit nutzen zum Dank, zur Bestandsaufnahme und Rechenschaft, zu kritischen, anregenden und hilfreich weiterführenden Gesprächen mit Ihnen allen. Deshalb ist das diesjährige Tagungsthema so weit gespannt „Die deutsche Sprache der 
Gegenwart - Perspektiven und Tendenzen". Es umreift das Arbeitsgebiet des Instituts vom ersten Tage an.

Die Ankündigung der Tagung hat, wie wir sehen, große Resonanz gefunden. 600 Germanistinnen und Germanisten sind aus 25 Ländern nach Mannheim gekommen, viele von Ihnen kennen das Institut und waren schon öfter hier, aber auch viele, und das freut uns, sind das erste Mal bei solch einer Tagung.

Eine so stattliche Teilnehmerzahl ist bisher bei noch keiner großen Jahrestagung erreicht worden. Die großen Tagungen finden jeweils in den geraden Jahreszahlen statt. Das ungerade 1989 hätte eigentlich eine der kleineren Zusammenkünfte mit dem Charakter eines Symposions werden sollen. Ich danke Ihnen sehr für Ihren Besuch und für Ihre Zeit, beides sind uns das wichtigste Jubiläumsgeschenk. Auf diese Weise bekunden Sie Ihr Interesse am Institut für deutsche Sprache, an seiner Arbeit und an der mit der Jahrestagung verbundenen Möglichkeit, alte kollegiale Kontakte zu pflegen und neue zu knüpfen und damit das Kohärenzbewußtsein in unserem heute oft bedrohten geisteswissenschaftlichen Fach zu festigen.

Am 18. April 1964 ist das Institut für Deutsche Sprache als eine Stiftung des bürgerlichen Rechts in Mannheim gegründet worden. Der Wunsch, die deutsche Sprache in umfassender Weise außerhalb der Hochschule und ungestört vom Lehrbetrieb zu beobachten und zu beschreiben, Wörterbücher zu verfassen und die Sprache in Grammatiken zu systematisieren, ist alt. Leibniz hatte bereits eine Forschungs- und Pflegestätte der deutschen Sprache gefordert, in der er gern die historische Lautentwicklung, eine vergleichende Grammatik der Mundarten, eine Grammatik des Sprachgebrauchs, aber auch die Fachsprachen und die Namen hätte erforschen lassen. Lexikographie und Sprachpflege wollte er auch behandelt sehen. Doch zur Gründung einer solchen Institution ist es nicht gekommen. Später sind an einzelnen Hochschulen spezielle Arbeitsstätten für Wörterbücher oder Forschungsschwerpunkte entstanden, wie das auch heute noch etwa bei den von der Deutschen Forschungsgemeinschaft geförderten Sonderforschungsbereichen geschieht. Aber sieht man einmal vom Deutschen Sprachatlas in Marburg oder von der Arbeitsstelle des Grimmschen Wörterbuchs in Göttingen als Institutionen überregionaler Wirksamkeit ab, so gab es nach 1945 keine zentrale Einrichtung zur Erforschung der deutschen Sprache.

Die Mannheimer Gründung war von einem Ausschuß vorbereitet worden, dem die Hochschulgermanisten Rudolf Hotzenköcherle (Zürich), Karl Kurt Klein (Innsbruck), Friedrich Maurer (Freiburg), Hugo Moser (Bonn), Jost Trier (Emeritus in Münster) und Leo Weisgerber (Bonn) 
angehörten und außer ihnen Paul Grebe (Mannheim) als Leiter der Dudenredaktion, und Walter Hensen, der Vorsitzende der Gesellschaft für deutsche Sprache, die damals ihren Sitz in Lüneburg hatte. Schon die Namen der Ausschußmitglieder kennzeichnen die Situation an den Hochschulen, die vermutlich die meisten von Thnen nicht mehr kennen. Fünf der genannten Universitätsprofessoren sind Altgermanisten, historische Sprachwissenschaftler und Dialektologen - auf allen drei Gebieten vorzüglich ausgewiesen -, die - vermutlich auch von der Entwicklung im Ausland angeregt - sehen, daß die Erforschung der Gegenwartssprache an den Universitäten nicht länger vernachlässigt bzw. ignoriert werden darf. Sprachwissenschaft in der Germanistik bedeutete bisher historische Grammatik, die mit all ihren Facetten im Indogermanischen begann und im akademischen Unterricht bis zum Mittelhochdeutschen reichte, also zum Frühneuhochdeutschen kaum vordrang, von der neueren und neusten Zeit ganz zu schweigen. Auch in der zusammenfassenden, überblicksmäßigen Sprachgeschichte fanden die beiden letzten Jahrhunderte stets nur eine knappe Darstellung. Im Studium war die Beschäftigung mit den Mundarten der einzige Vorstoß in die Gegenwart, allerdings auch meist, um das Beharrungsvermögen historischer Verhältnisse zu zeigen. Daß ich mit dieser stenogrammartigen Darstellung Namen wie Hermann Paul, Otto Behaghel, Erich Drach, Wolfgang Stammler u.a. auslasse, möge nicht mißverstanden werden. Aber die Situation des akademischen Unterrichts kam ohne die Beschreibung und Analyse des gegenwärtigen Deutsch aus. Leo Weisgerber vertrat im Gründungsgremium nicht nur die allgemeine Sprachwissenschaft. Er hatte in Bonn den Schwerpunkt „Sprache und Gemeinschaft" gegründet, der von der Deutschen Forschungsgemeinschaft gefördert wurde und in der Verfolgung Humboldtscher Ideen die Überlegungen zur inhaltsbezogenen Sprachforschung entwickelte. Paul Grebe leitete die Dudenredaktion. Das Bibliographische Institut mit Sitz in Mannheim verfügte über Wortkarteien und eine Fachbibliothek zum neuen Deutsch. Zweimal war in Mannheim der Dudenpreis an Gelehrte, die sich besondere Verdienste um die deutsche Sprache erworben hatten, verliehen worden, 1960 an Leo Weisgerber und 1962 an Hans Glinz. Die Gesellschaft für deutsche Sprache, die Herr Hensen vertrat, gab die "Muttersprache" heraus, damals eine der wenigen Zeitschriften, die sich ausschließlich mit der Gegenwart befaßte. Die Bundesrepublik, die Schweiz und Österreich waren vertreten, die Ausweitung zur allgemeinen Sprachwissenschaft hatte man bedacht, und neben den Hochschullehrern saßen Vertreter der angewandten Sprachwissenschaft mit Publikationserfahrung in Wörterbuch und Zeitschrift.

Die Zielsetzung der Institutsgründung war die Dokumentation, Beschreibung und Erforschung der deutschen Sprache nach 1945. Daß damit die 
Aneignung der inzwischen vielfaltig expandierenden germanistischen und internationalen Linguistik und die Auseinandersetzung mit ihr verbunden sein mußte, ahnte man vermutlich zunächst nicht. So entstand die Voraussetzung für neue Kontakte zur Auslandsgermanistik. Ihnen haben wir sehr zu danken, uns geholfen zu haben, allmählich die verhängnisvolle wissenschaftliche Isolation $2 u$ überwinden, in der wir uns nach dem Kriegsende gefangen sahen. Der Zeitpunkt der Gründung erweist sich aus der Rückschau als günstig. Das Jahr 1964 lag im wirtschaftlich konjunkturellen Aufschwung. Es begann die Gründerzeit im tertiären Bildungsbereich. $1965 \mathrm{nahm}$ die Ruhr-Universität Bochum als erste neue Hochschule die Vorlesungen auf, und bis zum Ende der 70er Jahre wurden 30 Universitäten gegründet; die bisherige Anzahl der Hochschulen hatte sich damit verdoppelt. An den meisten Neugründungen wurden germanistische Institute eingerichtet, die wohl auch auf die Signalwirkung aus Mannheim hin im linguistischen Studienteil das curriculare Pensum der historischen Sprachwissenschaft zugunsten der Gegenwartssprache relativierten.

Jost Trier hat in seiner Rede als dritter Dudenpreisträger am 19.4.1964 über das einen Tag vorher gegründete Institut gesprochen und dabei in aller Kürze nicht nur die Aufgaben skizziert, sondern auch Grenzlinien gezogen, die das Institut bis heute beachtet hat:

Denn:

1. Das Institut strebt keine gesetzgeberische Autorität an wie die Academia della Crusca, wie die deutschen Sprachgesellschaften des Barock oder die Academie Française.

2. Es werden die geschriebene und die gesprochene Sprache erforscht, aber nicht die Mundarten.

3. Das Institut wird nicht die Forschungsarbeiten aus den Universitäten herausziehen, sondern es will von seinem unabhängigen Standpunkt aus mit ihnen zusammenarbeiten und Forschungen koordinieren helfen.

4. Das Institut wird die Verbindung zum Institut für deutsche Sprache und Literatur an der Akademie der Wissenschaften in Berlin suchen. ( $\mathrm{DaB}$ wir diese inzwischen gefunden haben und von beiden Seiten pflegen wollen, hat uns das Grußwort von Herrn Schildt gezeigt, für das ich in unserer aller Namen herzlich danke.)

Eine Satzungsskizze nannte bereits das Kuratorium, den Vorstand und den wissenschaftlichen Rat als erste Gremien, allerdings ohne eine dezidierte Funktionsbestimmung zu geben. 
Wenn man der kleinen Arbeitsstelle von 1964, die im wesentlichen damit befaft war, ein maschinenlesbares Corpus von literarischen und expositorischen Texten der deutschen Gegenwartssprache zu erstellen, das heutige Institut gegenüberstellt, so, wie es sich Ihnen im Jahresbericht 1988 präsentiert, mit einem Jahresetat von rund 7.700.00 DM, 78 Mitarbeitern, darunter 44 Wissenschaftlern, rund 250 Buchveröffentlichungen, 4 Abteilungen, mit Kommissions-, Beirats- und Jahrestagungen und einem engen Netz von Vortrags-, Lehr-, Besuchs- und Beratungskontakten, dann ist man geneigt, sehr stolz auf das Erreichte zu sein. Im plakativen Bild der Statistik ergäbe sich zwischen 1964 und 1989 ein Streckenzug mit steilem Winkel. Doch die nähere Betrachtung zeigt ein anderes Bild mit Höhenunterschieden und Unterbrechungen. Ich will weder versuchen, die Geschichte des Instituts an seinen Forschungsarbeiten, noch an den Wechselbädern seiner wirtschaftlichen Rahmenbedingungen zu demonstrieren; aber ich möchte drei Erfahrungen aufzeigen, aus denen man den Gewinn einer allgemeinen Lehre ziehen könnte.

\section{Projekt und Etat}

Die finanzielle Ausgangssituation war 1964 für das junge Institut alles andere als verheißungsvoll; denn außer einem von der ThyssenStiftung großzügig zur Verfügung gestellten Startkapital von 160.000 DM und kleineren Beträgen der Kultusministerien von Baden-Württemberg und Nordrhein-Westfalen, des Ministeriums für gesamtdeutsche Fragen und von privater Seite zuzüglich der durch die Stadt Mannheim getragenen Miete für die Diensträume gab es keine in einem staatlichen Etat verankerte, im Rhythmus eines Haushaltsplanes wiederkehrende Existenzgrundlage, die eine längerfristige Planung gesichert hätte. Umso mehr muß man heute den Mut oder die Gelehrten häufig nachgesagte naive Unbeirrbarkeit bewundern, mit denen der einmal eingeschlagene Weg weitergegangen wurde, in den Wirbel heftigster Turbulenzen geriet, und schließlich doch langsam, aber erst vom 13. Jahr der Institutsgeschichte an zum heutigen Stand geführt hat. Das jahrelange und hartnäckige Bemühen um eine feste staatliche Etatisierung ging - selbst in der konjunkturell guten Zeit - schleppend voran - diametral entgegengesetzt zum Tempo der Ideen, die das Institut entwickelte und in immer neuen Projekten durchzuführen, einzuwerben oder als Auftrag zu übernehmen bereit war. 1974 und 1975 klaffte die Schere zwischen etatisierten Plan- und zeitlich limitierten Projektstellen besonders weit auseinander. Das Institut hatte damals seinen größten Personalstand, nämlich 135 Mitarbeiter, von denen nur 33 auf einer Planstelle saßen. Ich nenne diese Situation aus mehreren Gründen: einmal dürfen Engagement und Begeisterung 
nicht die Rahmenbedingungen im Sturm überrennen, und zum anderen ist es außerordentlich mühevoll und mißlich gewesen, in immer neuen Begründungs- und Rechtfertigungsanträgen darzulegen, weshalb ein Institut nötig ist, das die Landessprache erforschen und pflegen will, also das Kommunikationsmittel, welches gesellschaftliches Leben überhaupt erst möglich macht. Genehmigungsverfahren für große technische Einrichtungen, die dem Laien, der sie zu entscheiden hat, bestimmt oft völlig unverständlich sind, kommen ohne diese langwierige Rechtfertigungsredundanz aus. Und die weitere Problematik scheint mir in der Forschungskategorie „Projekt" zu liegen, die wegen der zeitlichen Begrenzung von den Stiftungen finanziert wird. Projekte sind in Einzelfällen unbestritten sinnvoll. Doch die Kumulierung ist, wie sich im Institut zeigte, nicht gut; denn ein Forschungsgebiet braucht die ständige Beleuchtung einer Flutlichtanlage und nicht den bunten Knalleffekt von Leuchtraketen, die nach steilem Aufstieg oft vor der garantierten Leuchtzeit verlöschen, weil die guten Mitarbeiter nach der Halbzeit das erste Angebot einer längerfristigen Tätigkeit annehmen und das Projekt verlassen.

\section{Das Haus}

Damit meine ich sowohl die finanzielle Existenzgrundlage als auch die Behausung. 1977 wurde das Institut in die sogenannte Blaue Liste aufgenommen, d.h. in das Verzeichnis der Forschungseinrichtungen, die gemeinsam von Bund und Ländern gefördert werden. Bei den Zuwendungsgebern, dem Bundesministerium für Forschung und Technologie und dem Ministerium für Kunst und Wissenschaft des Landes Baden-Württemberg, möchte ich sehr für die etatisierte Förderung danken, die den Haushalt des Instituts konsolidiert hat und eine kontinuierliche progressive Planung erlaubt. Der Dank gilt nicht nur dem unerläßlichen finanziellen Aufwand, sondern auch dem förderlichen Rat und der verständnisvollen Kritik, die wir von den Ministerialvertretern im Kuratorium und in vielen Einzelgesprächen empfangen haben. Für die unsichere Zeit des abenteuerlichen, zum Glück seit etwa 12 Jahren überwundenen Prae-Etatismus danke ich dem Land Nordrhein-Westfalen, dem GoetheInstitut, der Deutschen Forschungsgemeinschaft, der Thyssen-Stiftung und der Stiftung Volkswagenwerk für ihre Großzügigkeit und ihre lebensrettende Hilfe, die zweimal die erwogene Schließung des Instituts verhindert hat, und nicht zuletzt für die vielfaltigen Hilfen von Mannheimer Seite, vom Bibliographischen Institut und von der Stadt und ihrem Oberbürgermeister.

Nach dem Dank einen Satz mit "doch" zu beginnen, könnte als charakterlose, weil grenzenlose Habgier ausgelegt werden, wenn ich für das „doch” 
nicht die guten Argumente des Wissenschaftsrates im Köcher hätte, die - obwohl nicht neuesten Datums - nichts an Aktualität verloren haben. 1971 hatte sein zweites Gutachten empfohlen, das Institut als mittelgroße Forschungseinrichtung mit 80-100 Planstellen auszustatten. Wenn wir also in Anbetracht der finanziellen Engpässe von Bund und Land mit bescheidener Zurückhaltung, aber in Anbetracht des kompetenten Gutachtens und unserer künftigen Planung mit Beharrlichkeit um neue Stellen bitten, dann nicht, um das Institut zu erweitern, sondern um es der 1971 entworfenen Konzeption, die noch immer von überzeugender Gültigkeit ist, entgegenzuführen.

In diesem Gutachten hatte der Wissenschaftsrat auch den Bau eines eigenen Gebäudes empfohlen. Der Vorstand bemüht sich, in Rücksprache mit der Stadt und den beiden Ministerien eine Lösung zu finden, das

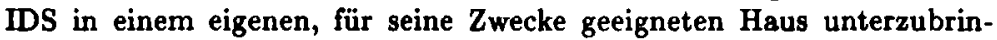
gen. Doch die sich anbahnende Möglichkeit droht wieder in die Ferne zu rücken. Liegt dieser schleppende Gang der Entwicklung tatsächlich nur an der Knappheit der Resourcen, die eine andere Prioritätensetzung fordert? Oder messen die Politiker der Erforschung und Pflege der deutschen Sprache nicht die Bedeutung bei, die ihr zukommt? Ein Haus der Geschichte wird gebaut für 79 Millionen, um die Identität der Bürger mit ihrem Staat zu erleichtern. Für ein Haus der deutschen Sprache stehen die veranschlagten 5 Millionen Mark nicht so ohne weiteres zur Verfügung. Dabei gibt es eine klare Konzeption, die auf der Grundlage einer 25jährigen Erfahrung gewachsen, korrigiert und gereift ist: Grammatik, Lexik, Sprache und Gesellschaft, Dokumentation, Lautarchiv und Bibliothek sind Forschungsinstrumentarien, die dem In- und Ausland zur Verfügung stehen, die den Deutschunterricht an Schulen und Hochschulen fördern, die Tagungen ermöglichen, die den Stellenwert unserer Sprache in der Welt und besonders im Sprachenmosaik der europäischen Gemeinschaft bestimmen helfen. An Aufgaben und Zielen fehlt es nicht; aber doch offenbar am politischen Willen, etwas in die Tat umzusetzen, dessen Relevanz und Notwendigkeit man vielleicht wegen der vermeintlichen Selbst verständlichkeit dieser Sprache nicht einzusehen vermag. Wir beobachten diese bedenkliche Haltung auch bei den stellenweise rigorosen und unüberlegten Kürzungs- und Sparmaßnahmen, denen unser Fach in der Hochschulpolitik einiger Länder ausgesetzt ist. Ein Blick über die Landesgrenzen hinweg würde zeigen, daß andere Länder auf diesem Felde ganz anders verfahren; ich möchte stellvertretend nur Frankreich und Japan nennen. 


\section{Die Generationsfrage}

Eingangs habe ich auf eine Gelehrtengeneration hingewiesen, die die zurückgelegten $25 \mathrm{Jahre}$ bedeuten. Falls es an den deutschen Hochschulen einmal das Fach Wissenschaftsgeschichte geben sollte, dann dürfte die Darstellung der Linguistik und ihrer Entwicklung am Beispiel des Instituts für Deutsche Sprache nicht zu den leichten Aufgaben gehören. Die personelle Besetzung der wissenschaftlichen Mitarbeiterstellen ist in der Hauptsache, so wie wir sie heute vorfinden, zu Beginn der 70er Jahre erfolgt, und zwar mit Damen und Herren, die Linguistik studiert haben, deren Interesse vorwiegend in der Synchronie liegt, d.h., die in ihren sprachwissenschaftlichen Studien und ihrer Einschätzung der germanistischen Linguistik eine relativ homogene Gruppe bilden - soweit man als Germanistische Gruppe überhaupt zur Homogenität neigt - und die weit vom Wissenschafts- und Theorieverständnis der Gründungsväter entfernt sind. Sie sind sozusagen die Gründungsenkel oder sogar -urenkel. Der Vergleich hinkt; aber ich meine, die Acceleration in der Linguistik ist so rasch vorangegangen, daß die Generationsabstände mentalitätsmäßig sehr viel kürzer geworden sind. Innerhalb des Instituts gibt es wohl Meinungsverschiedenheiten, aber kaum Generationsprobleme. Die treten erst auf, wenn die zwischen Gründern und Mitarbeitern liegende Generation janusgesichtig zwischen gestern und morgen als Kurator, Beiratsoder Kommissionsmitglied auftritt und Kritik zu äußern wagt. Aber das Generationsproblem wächst und arbeitet weiter; und zwar in doppelter Sicht. Die Gründertöchter und -söhne müssen sich täglich im lehrenden, beratenden und prüfenden Zusammenleben mit den für sie immer jünger werdenden Studenten bewähren und wenn sie dies ernsthaft tun, haben sie die Möglichkeit, im hohen Alter jünger zu sein als mancher InstitutsMitarbeiter, der nicht gemerkt hat, dab er in der Geborgenheit des Instituts alt geworden ist, weil ihn nicht die Jahre, sondern die Monotonie der hohen Spezialisierung frühzeitig hat welken lassen. Und auf der anderen Seite hält die Altershomogenität der Mitarbeiterschaft die Stellen naturgemäB lange besetzt, so daß eine Verjüngung durch Eintritt Nachwachsender kaum möglich ist und die Gefahr eines weiteren künftigen Generationensprunges besteht. Dieses Problem wird es in jeder Disziplin mit hoher Expansions- und Entwicklungsgeschwindigkeit geben. In den Geisteswissenschaften ist es in der Linguistik besonders ausgeprägt, wir können dies auch an den verschlungenen Wegen beobachten, die das IDS und die Deutsche Gesellschaft für Sprachwissenschaft umeinander herumgehen. Ich meine, diese Differenzen sollten behoben werden; denn wenn alle Sprachwissenschaftler und Linguisten im Hinblick auf die gemeinsame Arbeitsgrundlage, eben die Wissenschaft von der deutschen Sprache, zum Überwinden von Vorurteilen und zur offenen Diskussion 
bereit sind, kann jede Arbeit nur gefördert werden. Die Überbrückung von diesen jäh aufgebrochenen Generations- und Mentalitätsgräben wird der Zersplittung des Faches entgegenwirken. Es handelt sich hier keineswegs nur um eine Frage des Umgangs miteinander, sondern um ein fachstrukturelles Problem, das man mit Thomas Bernhard neinfach kompliziert" nennen kōnnte.

Die Etatisierung und die Projekte, die Behausung des Instituts und die Generationenfrage habe ich vor dem Hintergrund der letztlich bis heute erfolgreichen Institutsgeschichte als Problemkreise genannt, die inhaltlich wohl nicht zur Textsorte der Jubiläumsrede passen, aber deren Diskussionsdringlichkeit - wie ich meine - uns weiterbringen könnte: doch mit dem Blick auf die unbestreitbaren Erfolge und Verdienste möchte ich textsortengerecht mit einem Dank schließen:

an Herrn Moser, den Ehrenpräsidenten und Gründer, der als erster Präsident dem Institut 17 Jahre vorgestanden hat, und an Herrn Rupp, seinen Nachfolger und meinen Vorgänger, der dem Institut ein wetterfestes Satzungsgerüst geschenkt hat, an alle Kuratoren, Beirats- und Kommissionsmitglieder, an die bisherigen 3 Direktoren, Herrn Engel, Herrn Stickel und Herrn Wimmer für ihr Engagement und ihre große Arbeitsleistung, an alle wissenschaftlichen und nichtwissenschaftlichen Mitarbeiter des Instituts und nicht zuletzt an Sie alle als unsere unentbehrlichen kollegialen Gesprächspartner und Kritiker. 